\title{
The biology and function of exosomes in cancer
}

\author{
Raghu Kalluri
}

Department of Cancer Biology, Metastasis Research Center, University of Texas MD Anderson Cancer Center, Houston, Texas, USA.

\begin{abstract}
Humans circulate quadrillions of exosomes at all times. Exosomes are a class of extracellular vesicles released by all cells, with a size range of 40-150 nm and a lipid bilayer membrane. Exosomes contain DNA, RNA, and proteins. Exosomes likely remove excess and/or unnecessary constituents from the cells, functioning like garbage bags, although their precise physiological role remains unknown. Additionally, exosomes may mediate specific cell-to-cell communication and activate signaling pathways in cells they fuse or interact with. Exosomes are detected in the tumor microenvironment, and emerging evidence suggests that they play a role in facilitating tumorigenesis by regulating angiogenesis, immunity, and metastasis. Circulating exosomes can be used as liquid biopsies and noninvasive biomarkers for early detection, diagnosis, and treatment of cancer patients.
\end{abstract}

\section{What are exosomes?}

Exosomes are 40-150 $\mathrm{nm}$ extracellular vesicles (EVs) released by all cell types (1-3). The definition of the term exosomes has evolved with time (2), and in this review, exosomes are defined as small EVs with a multivesicular endosomal origin (2). This definition is challenged by the experimental difficulties associated with the study of exosomes, as the enriched exosome preparations that are obtained using current methodologies may not strictly discriminate from microvesicles shed from the budding of the plasma membrane, independent of intracellular multivesicular endosomes $(2,4,5)$. The definition of the term exosomes will likely continue to evolve over time in the scientific literature with ongoing efforts to better track their endocytic origin and distinguish them from other microvesicles (6).

Exosomes are highly heterogeneous (6) and likely reflect the phenotypic state of the cell that generates them (Figure 1). Similar to cells, exosomes are composed of a lipid bilayer and, at any given point, can contain all known molecular constituents of a cell, including proteins, RNA, and DNA (7-9). Notably, as a result of repeated invagination of the lipid bilayer membrane during their biogenesis, the orientation of the lipid bilayer of exosomes mirrors that of the cells they originate from.

\section{What are the functions of exosomes?}

The precise function of exosomes remains unknown. Early hypotheses favor the notion that exosomes may function as cellular garbage bags that expel excess and/or nonfunctional cellular constituents. Additionally, exosomes are EVs of endocytic origin and are likely generated to recycle cell surface proteins and, in turn, modulate outside-in signaling $(3,10)$. While this implies that exosomes contain specific constituents that are present in excess in a given cell, this has not been explicitly encountered or

Conflict of interest: MD Anderson Cancer Center and R. Kalluri hold patents in the area of exosome biology and are licensed to Codiak Biosciences Inc. MD Anderson Cancer Center and R. Kalluri are stock equity holders in Codiak Biosciences Inc. R. Kalluri receives research support from Codiak Biosciences Inc. and serves as a member of the board of directors

Reference information: / Clin Invest. 2016;126(4):1208-1215. doi:10.1172/JCI81135. proven. Whether particular cellular constituents are specifically shuttled into exosomes via an organized mechanism or whether exosome packaging is just a random process remains unknown. The content and numbers of exosomes generated likely change depending on whether cells are experiencing different stressors or stimuli, and exosomes made from the same cells can contain distinct constituents (Figure 1; refs. 2, 8, 11). It is possible that the content of exosomes is a reflection of what the cell is experiencing; however, some proteins are consistently associated with exosomes, implicitly arguing for some degree of specificity in trafficking proteins into exosomes (Figure 1). It is unclear which types of proteins might be enriched in exosomes, but emerging evidence suggests that some of these proteins are associated with exosome biogenesis. Plasma membrane anchors may play an important role in enriching specific proteins associated with exosomes (5). Critically, the discovery that exosome contents can be transferred to a recipient cell via fusion to mediate phenotypic alterations supports the notion that exosomes are dynamic mediators of intercellular communication $(8,12)$.

Conservatively, normal human blood is estimated to contain about 2,000 trillion exosomes, and the blood of cancer patients is estimated to contain about 4,000 trillion exosomes (13-15). The diseased organs and the abnormal cells within them generate even more exosomes $(13,16)$. The underlying causes of this increase in exosome generation remain unknown, but it is speculated to be due to altered cellular physiology. While the rate of exosome production and the heterogeneity of exosomes derived from cells in normal physiological conditions can be difficult to determine in vivo, many in vitro studies indicate that virtually all cell types can produce exosomes, that exosomes are heterogeneous, and that heterogeneity may be enhanced in pathological conditions such as cancer.

\section{How are exosomes generated?}

The generation of exosomes via inward budding of the plasma membrane to form intracellular endosomes was first reported in sheep reticulocyte maturation as a means for cell surface protein turnover (10). Further invagination of the intracellular endosomes generates multivesicular bodies (MVBs) containing vesicles with a diameter of 40-150 nm. MVBs then fuse with lysosomes 
A

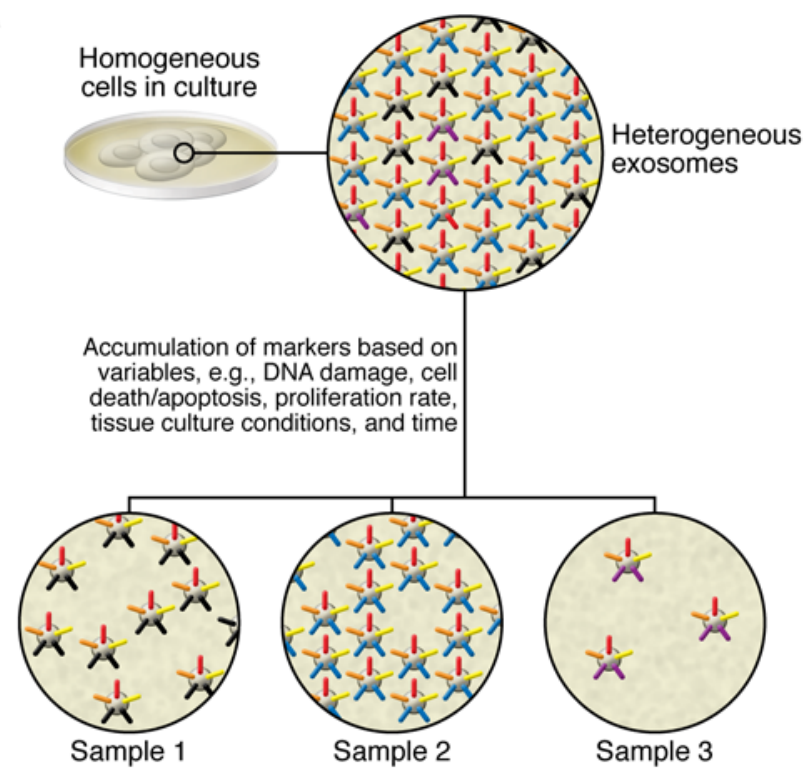

B

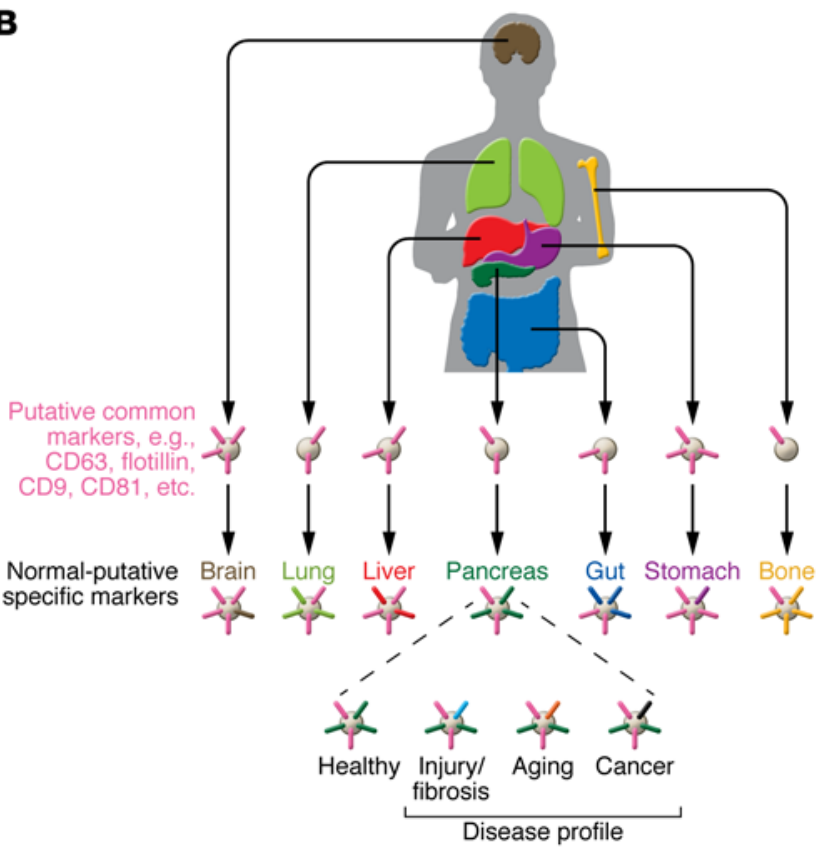

for degradation of their contents or fuse with the plasma membrane, releasing their contents into the extracellular space in the form of exosomes $(10,17)$. This process, termed exosome biogenesis, distinguishes exosomes from EVs that emerge via outward budding of the plasma membrane, apoptotic bodies, or necrotic blebs of the plasma membrane. Exosome biogenesis is observed in immune cells, mesenchymal stem cells, fibroblasts, neurons, endothelial cells (ECs), and epithelial cells. Similar size vesicles may also arise from diverse types of intraluminal vesicles (ILVs); however, specific markers to distinguish these vesicles are lacking; therefore, the generic term of EVs is currently being employed (4).

A core component of exosome biogenesis involves the endosomal sorting complexes required for transport complexes (ESCRT complexes), although an ESCRT-independent pathway involving ceramides for exosome biogenesis has also been reported (18).
Figure 1. Exosome biology and heterogeneity is a reflection of the origin and status of the originating tissue or cell at the time of exosome generation. (A) In culture, the same cell type can produce exosomes with distinct nucleic acids and proteins above the baseline of some common protein markers. Exosome production can be dynamic, with the same cell shedding exosomes with different compositions based on their health status. (B) It has been assumed that exosomes are cellular trash bags for elimination of excess proteins, but many studies have shown that exosomes often exhibit certain specific markers, irrespective of the organ or cellular source. This suggests that certain proteins are transported to the exosomes with some degree of specificity. Additionally, distinct differences have been identified in the RNA and protein species present in exosomes, likely reflecting cellular and organ specificity. This second layer of complexity is dynamic and can change with the evolving status of the organ and cells within. Here, the pancreas is provided as an example, but this dynamism in exosomal contents presumably holds true for all organs. Therefore, exosome heterogeneity is a much more complex phenomenon than cellular heterogeneity and can be fleeting depending on stimuli and the functional status of the organ.

The multiprotein complexes composed of ESCRT-0, -I, -II, and -III - together with accessory proteins (ALIX, VPS4, and VTA1) - localize on the cytoplasmic side of the endosomal membrane, participate in the sorting of proteins into ILVs, and involve ubiquitination of target proteins (19). This multiprotein complex recruits deubiquitinating enzymes and further sorts proteins into the ILVs. Other proteins associated with the endocytic pathway have also been recognized as important for exosome biogenesis, including annexin A2, RAB5/7/27, and TSG101, and Ostrowski et al. (20) further refined the specific roles of Rab27 isoforms in the exosomal pathway. The rate of exosome biogenesis is largely unknown and likely differs between cell types and their physiological or pathological status. While RNAs (mRNA and $\mathrm{miR}$ ) are conceivably incorporated into exosomes during the formation of ILVs, the mechanism of how genomic DNA gets into exosomes remains unknown and highlights an intriguing possibility for yet unknown additional regulatory pathways associated with exosome biogenesis.

\section{What do exosomes contain?}

Upon release from the cell surface, exosomes possess the capacity to fuse with the plasma membranes of recipient cells to deliver their contents into the cytoplasm. Alternatively, proteins present on the surface of the exosomes can engage cell surface receptors on recipient cells to induce intracellular signaling (19, $21,22)$. Thus, the contents of exosomes are a critical determining factor in their effects.

The lipid composition of exosomes includes cholesterol, sphingomyelin, hexosylceramides, phosphatidylserine, and saturated fatty acids (19), all of which are components of the plasma membranes. The proteome of exosomes includes endosomal, plasma, cytosolic, and nuclear proteins (23). Proteins enriched in exosomes include proteins associated with membrane transport and fusion, including Rab GTPases and annexins, as well as proteins involved in exosome biogenesis (ESCRT complex, ALIX, TSG101). Exosomes are also enriched with heat shock proteins (HSP70, HSP90), integrins, tetraspanins (CD9, CD63, CD81, and CD82) $(3,19,24)$, MHC class II proteins (3), epithelial cell adhesion molecules (EpCAM) (25), and members of the human epidermal receptor (HER) family $(26,27)$. Many exosomal protein con- 


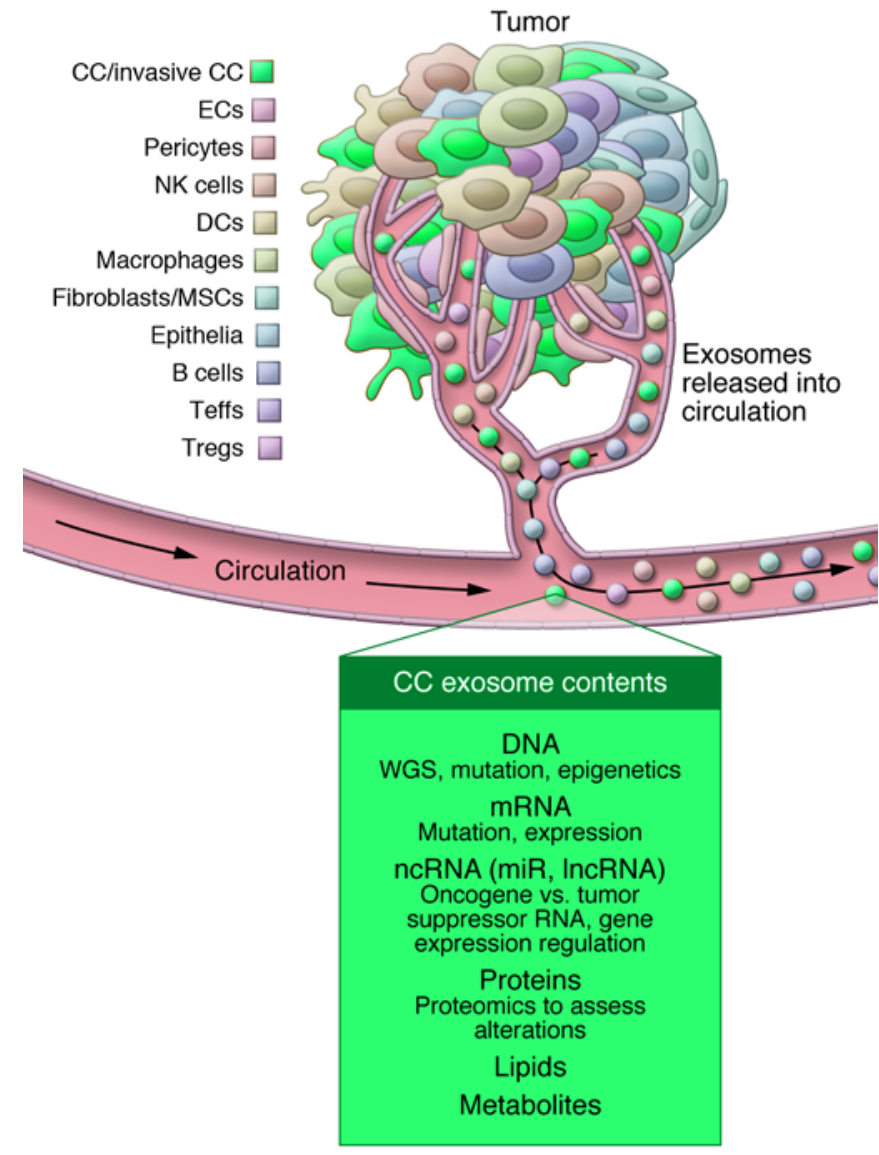

stituents likely reflect the common exosome biogenesis pathway, whereas other proteins may be enriched in exosomes as a result of enhanced levels in the cell of origin (Figure 1).

miR and mRNA were the first classes of nucleic acids identified in exosomes $(8,28,29)$. Subsequently, all other RNA species were identified in exosomes including transfer RNAs (tRNAs), long noncoding RNAs (lncRNAs), and viral RNA $(29,30)$. Interestingly, the enrichment of specific mRNA in exosomes may in part implicate a "zipcode" sequence in this process (31). Different types of RNAs, including mRNA and noncoding RNAs such as miRs and lncRNAs $(8,14,32)$, in exosomes are functional and can impact the transcriptome of recipient cells $(8,14,33,34)$. The functional impact of nucleic acids in exosomes for cell-to-cell communication in the setting of embryonic and organ development, normal physiology, and various pathologies are still emerging. Balaj et al. (9) demonstrated the presence of small fragments of single-stranded DNA in exosomes, and Kahlert et al. (7) identified large fragments of genomic, double-stranded DNA (>10 kb) encompassing all chromosomes inside the exosomes.

\section{Functions of exosomes in cancer}

In cancer, exosomes have been largely described as promoters of tumor progression $(14,35,36)$; however, it is conceivable that exosomes also possess antitumor functions and act to restrain disease progression. To tease out the potential beneficial aspects of exosomes in tumors, further studies are required to interrogate the complexity and functional heterogeneity of exosomes. The roles
Figure 2. Tumor-associated and circulating cancer-derived exosomes are a heterogeneous population that generates a unique tumor nanoenvironment (TNE). Tumors are a composite of cancer cells (CCs) with different genetics and phenotypes, stromal cells (including immune cells), mesenchymal cells (such as mesenchymal stem cells [MSCs] and fibroblasts), $\mathrm{ECs}$, and stromal elements (such as ECM components). Immune cell subtypes can be found in the tumors and include DCs, B cells, Teffs, Tregs, NK cells, and macrophages. All of these cells shed exosomes, contributing to TNE diversity. These exosomes likely participate in cell-to-cell communications within the tumor microenvironment and contribute to the heterogeneity of circulating exosomes. Exosomes derived from the serum or plasma can yield DNA for whole genome sequencing (WCS) and the identification of gene mutations and deletions. Additionally, exosomes allow for the analysis of mRNA; noncoding RNA (ncRNA), including miR and IncRNA; proteins; lipids; and metabolites.

of exosomes in pathological remodeling of organs other than cancer or in pregnancy are being unraveled (11); however, the normal, physiological functions of exosomes in tissue homeostasis remain unknown, reflecting the current difficulty of studying normal physiological function of exosomes. Exosomes and their contents in particular have emerged as a potential source of information to detect cancer and to provide information on potential regulatory drivers of tumor progression and metastasis.

Exosomes in the detection of cancer. Exosomes have been detected in many biological fluids, including urine, semen, saliva, amniotic fluid, cerebrospinal fluid, bile, ascites, tears, breast milk, and blood $(2,11,37,38)$. Cancer exosomes may serve as a liquid biopsy to aid in the diagnosis of malignancies, including prostate, pancreas, breast, and ovarian cancers; glioblastoma; and melanoma (13, 39-43). Exosome concentration are reportedly elevated in the systemic circulation of patients with ovarian, breast, and pancreatic cancer $(13,16)$. Specific markers associated with cancer exosomes may enhance their enrichment and may be useful for diagnosis when harvested from a heterogeneous population of exosomes in body fluids (Figure 2). Many in vitro and preclinical studies have enhanced our understanding of exosome content and its potential utility to detect and monitor cancer. While lipids and metabolites in cancer exosomes may also offer unique insights in the detection and biology of cancer, more precise knowledge is evolving with respect to the utility of proteins and nucleic acids in exosomes (Figure 2).

Nucleic acids in cancer exosomes. Unique nucleic acids (mutant mRNA such as the epidermal growth factor receptor EGFRvIII variant) contained in exosomes could potentially serve as reliable biomarkers for glioblastoma (43). Enriched and specific miRs in exosomes may also inform diagnosis and serve to monitor the progression of cancer $(16,44-48)$. For example, exosomal miR-21 is increased in the serum of patients with esophageal squamous cell carcinoma and correlates with advanced disease stage (45). Exosomal miR-141 levels in the serum of patients with prostate cancer discriminates those with metastatic disease from those with localized disease (47). Analyses of exosomes derived from urine revealed a miR profile that may enable the detection of urothelial carcinoma of the bladder (46).

DNA in exosomes may provide information about cancerspecific mutations $(7,49)$. Whole genome sequencing revealed that exosomes in the serum of pancreatic cancer patients contained the entire genomic double-stranded DNA encompassing 
all chromosomes. Additionally, driver mutations associated with pancreatic ductal adenocarcinoma (PDAC) were identified in this exosomal DNA (7). Other research groups validated this discovery in their subsequent publications (49-51). An opportunity for whole genomic sequencing from serum exosomes of cancer patients has the potential to inform diagnosis and predict therapy outcomes.

Proteins in cancer exosomes. Exosome proteins likely reflect their cellular origin and may also aid in the detection of cancer. Intercellular transfer of oncoproteins by exosomes may play a role in facilitating tumorigenesis $(52,53)$. In stage 3 and stage 4 melanoma patients, circulating exosome-associated MET (also known as a hepatocyte growth factor receptor) and phosphorylated MET (Tyr1349) are increased compared with healthy controls (36). Exosome proteins may also be useful in detecting pancreatic cancer. Costa-Silva et al. (35) reported that macrophage migration inhibitory factor (MIF) is elevated in circulating exosomes of PDAC patients with disease progression after diagnosis when compared with healthy control subjects or PDAC patients with no evidence of disease 5 years after diagnosis and treatment. The cell surface proteoglycan glypican-1 (GPC-1) was detected on exosomes harvested from the serum of patients with pancreatic cancer and breast cancer (13). GPC-1 was detected in both early and late stages of pancreatic cancer, with negligible levels detected on exosomes from the serum of healthy donors (13). GPC-1 on exosomes discriminated patients with PDAC from those with benign pancreas diseases, such as chronic pancreatitis (13).

Exosomes are key components of the tumor microenvironment. Tumor heterogeneity encompasses genomic heterogeneity among neoplastic cells in the tumor, as well as non-cancer cell tumor microenvironment heterogeneity. Diverse composition of immune cells, mesenchymal cells, and acellular constituents contribute to the functional heterogeneity of tumors. Tumor nanoenvironment (TNE) heterogeneity is emerging as another layer of complexity in the tumors. The TNE involves EVs of various sizes, as well as apoptotic bodies. Exosomes derived from cancer cells may participate in generating a protumorigenic or antitumorigenic milieu by manipulating host stromal responses. Exosomes from stromal cells may promote or limit cancer progression in a contextdependent manner.

Exosomes and tumor immunity. Early studies elucidating the biological functions of exosomes have implicated them in the regulation of adaptive immunity $(3,54)$. The immunological activities of exosomes in tumors are likely complex and dynamic, ranging from modulation of tumor antigen presentation to polarization of tumor immunity $(55,56)$. While exosomes have emerged as critical mediators of immune/cancer cell communication, their anti- and protumorigenic roles are unclear, possibly reflecting the functional heterogeneity of exosomes in the tumor microenvironment (Figure 2). Exosomes from DCs can activate T and B cells, and cancer cellderived exosomes may serve as a source of tumor antigens that can be presented to the activated $\mathrm{T}$ cells (57-62). Cancer cell-derived exosomes may also directly activate NK cells via presentation of the stress protein HSP7O (63), and mast cell-derived exosomes may indirectly activate $\mathrm{T}$ and $\mathrm{B}$ cells via DC differentiation (64). While the reported immune activities of exosomes support their role in promoting antitumor immune responses, exosomes may also aid in immune evasion by impairing DC maturation via induction of IL-6 expression in BM dendritic precursor cells (65). Exosomes from cancer cells can also inhibit NK cell proliferation and cytotoxic functions via downregulation of NK group 2, member D (NKG2D) (66). Cancer cell-derived exosomes can impact $\mathrm{T}$ cell biology by inducing $\mathrm{T}$ cell apoptosis (67). Fas ligand (FasL) on cancer cell-derived exosomes induces apoptosis of $\mathrm{Fas}^{+} \mathrm{T}$ cells (67). Cancer cell-derived exosomes may also suppress $\mathrm{T}$ cell receptor (TCR) activity $(68,69)$ and regulate the transcriptome of regulatory and effector $\mathrm{T}$ cells (Tregs and Teffs) (70). TGFß1 in cancer cell-derived exosomes is reported to induce Tregs (71). Collectively, such effects of exosomes are speculated to result in antitumor immune responses. A better understanding of the immune functions of exosomes in tumors may suggest novel and efficient antitumor therapies. Ongoing clinical efforts include the treatment of cancer patients (non-small-cell lung cancer) with DC-derived exosomes (dexosomes) to activate the antitumor immune response (72).

Exosomes in tumor angiogenesis. Exosomes also participate in the regulation of pathological angiogenesis, including tumor angiogenesis (73). Uptake of cancer cell-derived exosomes by ECs, particularly in response to enhanced exosome production due to intratumoral hypoxia, stimulates angiogenesis $(43,74-76)$. This is achieved in part via stimulation of the proangiogenic secretome of ECs (77). Angiogenic programs launched by hypoxia-induced cell signaling in cancer cells can be influenced by exosomes. The impact of tumor-derived exosomes on vascular remodeling may not only affect tumor growth, but metastasis as well. For example, melanoma-derived exosomes induce vascular leakiness at the sites of metastases and influence the provasculogenic phenotype of recruited BM progenitors (36). Cancer cell-derived exosomes may also impair the structural integrity of ECs. The uptake of cancer cell-derived exosomal miR-105 by ECs downregulates the tight junction protein $\mathrm{ZO}-1$, thereby enhancing vascular permeability and metastatic dissemination (78).

Exosomes and cancer-associated fibroblasts. Exosomes may also significantly impact the tumor microenvironment by the activation of fibroblasts, facilitating tumor angiogenesis and tumor immunity. Specifically, cancer cell-derived exosomes from prostate cancer cells promote activation of myofibroblasts (79). Cancer cell-derived exosomes induce normal lung fibroblast activation (myofibroblast differentiation) in vitro (80) and promote acquisition of myofibroblast-like features in mesenchymal stem cells derived from adipose tissue (81). These studies support the hypothesis that stimulation by cancer cell-derived exosomes helps cancer-associated fibroblasts (CAFs) to acquire protumorigenic properties. Conversely, CAF-derived exosomes may also promote cancer progression. Hu et al. demonstrated that CAF-derived exosomes contribute to chemoresistance of colorectal cancer stem cells (82), and Boelens et al. implicated fibroblast-derived exosomes in expanding therapy-resistant breast cancer cells (83).

Regulation of tumor growth and metastasis by exosomes. The effects of stromal or cancer cell exosomes on recipient cells likely involve reciprocal signaling, which may or may not collectively result in a net tumor-promoting effect. While the vast majority of investigations report on the protumorigenic, proinvasive, and prometastatic effects of cancer cell-derived exosomes, it is important to keep in mind that stromal-derived exo- 


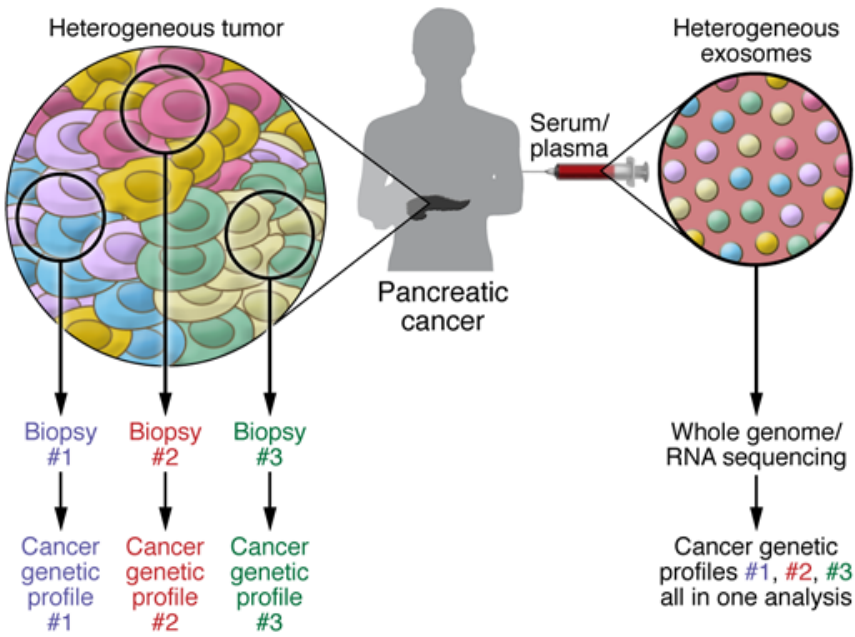

somes may have different and perhaps opposite functions from cancer exosomes. Cancer exosomes have been implicated in the conversion of benign epithelial cells into malignant cells, the remodeling of the extracellular matrix (ECM) to promote cancer cell invasion, the induction of invasive phenotypes in recipient cells, and the modulation of the metastatic site to enhance metastatic disease, as detailed below.

Exosomes and tumorigenesis. Cancer cell-derived exosomes have been implicated in determining the tumorigenic potential of epithelial cells $(14,21,84,85)$. Cancer exosome miR may also contribute to tumorigenesis, and cancer exosomes can mediate cell-independent miR biogenesis, a property not associated with normal exosomes. Cancer exosomes with RNA-induced silencing complex-associated (RISC-associated) miRs induce tumor formation by nontumorigenic mouse mammary cells (MCF10A cells). Notably, impairment of exosome-associated miR biogenesis via specific silencing of Dicer prevented the growth of MCF10A tumors (14).

Exosomes and tumor ECM remodeling. Exosome biogenesis is associated with invadopodia formation and multivesicular endosome docking (86). Release of exosome-derived proteinases may enhance ECM degradation associated with invadopodia maturation (86). Exosomes contain active proteases capable of ECM degradation, and they contribute to ECM remodeling by selectively and directly binding to the ECM-binding motif present on exosomal surface adhesion proteins (87). While it remains unclear how such exosomal contents are released, ECM remodeling by exosomes enhances fibroblast adhesion and migration (87).

Exosomes and metastasis. Exosomes can influence cells in the locale where they are produced as well as at distant sites, and the outcome of such influence may be site-specific and dependent upon the nature of interactions with the recipient cells. In tumors, the local impact of exosome-mediated cell signaling has generally been reported to promote invasive features of cancer cells associated with metastasis. These features include morphological changes associated with migratory functions (epithelialto-mesenchymal transition [EMT], cytoskeleton reorganization, and invadopodia formation), motility, and ECM and basement membrane remodeling activities. Invasion and colonization potential of cancer cells is enhanced when miR-200 from exo-
Figure 3. Sequencing of exosomal DNA and RNA can identify all driver and passenger mutations and deletions, providing information on actionable genetic defects associated with cancer. Tumors contain a heterogeneous mix of cancer cells. Clonal heterogeneity emerges when different sets of mutations and deletions drive different cancer cell clones, generating zones within tumors that contain unique sets of cancer cells with defined genetics. Therefore, tumor biopsies or portions cannot provide a view of the entire landscape of cancer-associated genetic defects. Analysis of cancer exosomes from the patient's blood can potentially overcome this limitation and offer genetic information reflecting the status of all the cancer cells in the tumor in order to account for tumor heterogeneity.

somes of invasive cancer cells are transferred to less invasive cancer cells (88), suggesting that cancer cell-derived exosomes can influence the invasive phenotype.

The impact of exosomes on the induction and/or maintenance of the EMT program in cancer cells is still emerging (89). It is conceivable that exosome contents differ when derived from epithelial-like versus mesenchymal-like cancer cells, and such exosomal heterogeneity may impact metastasis or resistance to therapy. A recent study implicated such "education" of benign tumor cells by malignant cancer cell-derived exosomes (12). Lineage-tracing experiments indicate that the malignant cancer cell-derived exosomes, when taken up by benign tumor cells, induce a conversion to the malignant phenotype (12). While the cancer cell-derived exosomes may promote metastasis by remodeling distant, metastasis-prone organs $(35,36)$, the precise and comprehensive action of exosomes at metastatic sites is not yet fully understood but may implicate specific integrin expression on exosomes (90).

\section{Function of exosomes in cancer therapies}

Tumor exosomes and resistance to therapy. Exosomes are emerging as a potential contributor to cancer progression, adding another dimension to the complexity of the tumor microenvironment. Therefore, it is conceivable that cancer exosomes could serve as a therapeutic target. Efforts to suppress horizontal transfer of miRs from cancer cells to ECs via exosomes attenuates angiogenesis and metastasis (91). Tumor stroma-derived exosomes have also been implicated in cancer chemoresistance $(34,82)$, supporting the notion that targeting specific functions of exosomes could enhance response to therapies. Exosomes may carry a defined set of miRs that transfer a resistance phenotype to sensitive cancer cells by altering cell cycle control and inducing antiapoptosis programs. Depletion of such exosomes limits invasive features of cancer cells (34). Transfer of fibroblast-derived exosomes to breast cancer cells may confer chemotherapy and radiation therapy resistance via activation of STAT1-dependent antiviral signaling and NOTCH3 signaling in cancer cells (83). Exosomes may also negatively impact chemotherapy treatment by shuttling out chemotherapeutic agents from target cancer cells $(92,93)$. Cisplatin and doxorubicin were found in cancer cell-derived exosomes in a posttreatment setting $(92,94)$. HER-2 ${ }^{+}$exosomes from HER-2-overexpressing breast cancer cells inhibit trastuzumab-induced antiproliferative activity (27); thus, removal of HER-2 ${ }^{+}$exosomes from the blood of patients with HER-2-overexpressing breast cancers might improve patient responses to trasuzumab (95). Exosome 
depletion from the blood of cancer patients may also minimize the immune tolerance potentially mediated by exosomes (96). While exosomal depletion may offer treatment benefits for cancer patients, more studies are required to understand the sum effect of exosome depletion on the body (97).

Exosomes as nanocarriers of anticancer therapies. The utility of exosomes as naturally derived delivery vehicles for therapeutic agents is being actively explored (98). Exosomes have been used to deliver miRs to specific targets $(99,100)$. Additionally, exosomes derived from immature DCs have been modified to contain protein fused to a peptide ligand that enables specific targeting of the exosomes to the brain via binding to acetylcoholine receptors on neuroendothelial and neuronal cells (99). This strategy enabled targeting of $\alpha$-Synuclein ( $\alpha$-Syn) using RNA interference molecules. This approach led to the reduction of intraneural $\alpha$-Syn protein aggregates in the brain (99). Delivery of let-7 miR by modified exosomes to specifically target $\mathrm{EGFR}^{+}$breast tumors reduced tumor burden in mice (100). Sophisticated engineering of exosomes may offer new therapeutic opportunities to control primary tumors and metastatic disease.

\section{The future of exosomes}

Understanding the precise physiological function of exosomes will be critical to determining their role in cancer. Questions remain as to whether it is necessary to generate exosomes to survive. Generating mice with specific and complete deletion of exosomes could begin to answer such questions. To achieve this, we need to learn more about the biology of exosomes and their precise biogenic mechanism. Generally, conclusions regarding the functional role of exosomes in facilitating cell-to-cell communication are based on in vitro experiments in isolated culture systems using concentrations of exosomes that are, at best, rough approximations and may not be physiologically relevant. Therefore, it is expected that in vivo studies to address the functional role of exosomes in cancer will be explored with increasing frequency.

Future studies will likely also focus on the potential heterogeneity of cancer exosomes and how this knowledge could benefit the understanding of clonal expansion of cancer cells and changes in clonal expansion in response to therapies. RNA and DNA sequencing of serum exosomes (liquid biopsies) will likely aid in the early diagnosis of cancer without the need for invasive procedures (Figure 3). Such technology coupled with proteomic analysis of exosomes could provide a window into the potential evolution of malignant disease in the body (Figure 3). Using exosomes to deliver drugs could offer new treatment modalities for our patients. This is the golden age of studies related to exosome biology, and future research promises to unravel many unknown actions of exosomes in health and disease.

\section{Acknowledgments}

The work on exosomes in the Kalluri laboratory is supported by Cancer Prevention and Research Institute of Texas, Metastasis Research Center, and NCI grant CA-195733. R. Kalluri wishes to thank Valerie S. LeBleu for her help in preparing this manuscript and also for the help in generating the schematic figures.

Address correspondence to: Raghu Kalluri, University of Texas MD Anderson Cancer Center, Department of Cancer Biology, 1881 East Road, Unit 1906, Houston, Texas 77054, USA. Phone: 713.792.8586; E-mail: rkalluri@mdanderson.org.
1. Kahlert C, Kalluri R. Exosomes in tumor microenvironment influence cancer progression and metastasis. J Mol Med (Berl). 2013;91(4):431-437.

2. Raposo G, Stoorvogel W. Extracellular vesicles: exosomes, microvesicles, and friends. J Cell Biol. 2013;200(4):373-383.

3. Thery C, Zitvogel L, Amigorena S. Exosomes: composition, biogenesis and function. Nat Rev Immunol. 2002;2(8):569-579.

4. Gould SJ, Raposo G. As we wait: coping with an imperfect nomenclature for extracellular vesicles. J Extracell Vesicles. 2013;2.

5. Shen B, Wu N, Yang JM, Gould SJ. Protein targeting to exosomes/microvesicles by plasma membrane anchors. J Biol Chem. 2011;286(16):14383-14395.

6. Kowal J, et al. Proteomic comparison defines novel markers to characterize heterogeneous populations of extracellular vesicle subtypes. Proc Natl Acad Sci U S A. 2016;113(8):E968-E977.

7. Kahlert C, et al. Identification of double-stranded genomic DNA spanning all chromosomes with mutated KRAS and p53 DNA in the serum exosomes of patients with pancreatic cancer. J Biol Chem. 2014;289(7):3869-3875.

8. Valadi H, Ekstrom K, Bossios A, Sjostrand M, Lee JJ, Lotvall JO. Exosome-mediated transfer of mRNAs and microRNAs is a novel mechanism of genetic exchange between cells. Nat Cell Biol. 2007;9(6):654-659.

9. Balaj L, et al. Tumour microvesicles contain retrotransposon elements and amplified oncogene sequences. Nat Commun. 2011;2:180.

10. Pan BT, Teng K, Wu C, Adam M, Johnstone RM. Electron microscopic evidence for externalization of the transferrin receptor in vesicular form in sheep reticulocytes. J Cell Biol. 1985;101(3):942-948.

11. De Toro J, Herschlik L, Waldner C, Mongini C. Emerging roles of exosomes in normal and pathological conditions: new insights for diagnosis and therapeutic applications. Front Immunol. 2015;6:203.

12. Zomer A, et al. In Vivo imaging reveals extracellular vesicle-mediated phenocopying of metastatic behavior. Cell. 2015;161(5):1046-1057.

13. Melo SA, et al. Glypican-1 identifies cancer exosomes and detects early pancreatic cancer. Nature. 2015;523(7559):177-182.

14. Melo SA, et al. Cancer exosomes perform cell-independent microRNA biogenesis and promote tumorigenesis. Cancer Cell. 2014;26(5):707-721.

15. Caradec J, et al. Reproducibility and efficiency of serum-derived exosome extraction methods. Clin Biochem. 2014;47(13-14):1286-1292.

16. Taylor DD, Gercel-Taylor C. MicroRNA signatures of tumor-derived exosomes as diagnostic biomarkers of ovarian cancer. Gynecol Oncol. 2008;110(1):13-21.

17. Harding C, Heuser J, Stahl P. Receptor-mediated endocytosis of transferrin and recycling of the transferrin receptor in rat reticulocytes. J Cell Biol. 1983;97(2):329-339.

18. Trajkovic K, et al. Ceramide triggers budding of exosome vesicles into multivesicular endosomes. Science. 2008;319(5867):1244-1247.

19. Colombo M, Raposo G, Thery C. Biogenesis, secretion, and intercellular interactions of exosomes and other extracellular vesicles. Annu Rev Cell Dev Biol. 2014;30:255-289.

20. Ostrowski M, et al. Rab27a and Rab27b control different steps of the exosome secretion pathway. Nat Cell Biol. 2010;12(1):19-30.

21. Zhang X, Yuan X, Shi H, Wu L, Qian H, Xu W. Exosomes in cancer: small particle, big player. J Hematol Oncol. 2015;8:83.

22. Mincheva-Nilsson L, Baranov V. Cancer exosomes and NKG2D receptor-ligand interactions: impairing NKG2D-mediated cytotoxicity and anti-tumour immune surveillance. Semin Cancer Biol. 2014;28:24-30.

23. Kowal J, Tkach M, Thery C. Biogenesis and secretion of exosomes. Curr Opin Cell Biol. 2014;29:116-125.

24. Simons M, Raposo G. Exosomes - vesicular carriers for intercellular communication. Curr Opin Cell Biol. 2009;21(4):575-581.

25. Runz S, et al. Malignant ascites-derived exosomes of ovarian carcinoma patients contain CD24 and EpCAM. Gynecol Oncol. 
2007;107(3):563-571.

26. Baran J, et al. Circulating tumour-derived microvesicles in plasma of gastric cancer patients. Cancer Immunol Immunother. 2010;59(6):841-850.

27. Ciravolo V, et al. Potential role of HER2-overexpressing exosomes in countering trastuzumab-based therapy. J Cell Physiol. 2012;227(2):658-667.

28. Ratajczak J, et al. Embryonic stem cell-derived microvesicles reprogram hematopoietic progenitors: evidence for horizontal transfer of mRNA and protein delivery. Leukemia. 2006;20(5):847-856.

29. Gusachenko ON, Zenkova MA, Vlassov VV. Nucleic acids in exosomes: disease markers and intercellular communication molecules. Biochemistry (Mosc). 2013;78(1):1-7.

30. Bullock MD, et al. Exosomal non-coding RNAs: diagnostic, prognostic adn therapeutic application in cancer. Non-Coding RNA. 2015;1(1):53-68.

31. Bolukbasi MF, et al. miR-1289 and "Zipcode"-like Sequence Enrich mRNAs in Microvesicles. Mol Ther Nucleic Acids. 2012;1:e10.

32. Crescitelli R, et al. Distinct RNA profiles in subpopulations of extracellular vesicles: apoptotic bodies, microvesicles and exosomes. J Extracell Vesicles. 2013;2.

33. Deregibus MC, et al. Endothelial progenitor cell derived microvesicles activate an angiogenic program in endothelial cells by a horizontal transfer of mRNA. Blood. 2007;110(7):2440-2448.

34. Chen WX, et al. Exosomes from drug-resistant breast cancer cells transmit chemoresistance by a horizontal transfer of microRNAs. PLoS One. 2014;9(4):e95240.

35. Costa-Silva B, et al. Pancreatic cancer exosomes initiate pre-metastatic niche formation in the liver. Nat Cell Biol. 2015;17(6):816-826.

36. Peinado $\mathrm{H}$, et al. Melanoma exosomes educate bone marrow progenitor cells toward a prometastatic phenotype through MET. Nat Med. 2012;18(6):883-891.

37. Keller S, Ridinger J, Rupp AK, Janssen JW, Altevogt P. Body fluid derived exosomes as a novel template for clinical diagnostics. J Transl Med. 2011;9:86.

38. Lasser C. Identification and analysis of circulating exosomal microRNA in human body fluids. Methods Mol Biol. 2013;1024:109-128.

39. Corcoran C, Friel AM, Duffy MJ, Crown J, O'Driscoll L. Intracellular and extracellular microRNAs in breast cancer. Clin Chem. 2011;57(1):18-32.

40. Li J, Sherman-Baust CA, Tsai-Turton M, Bristow RE, Roden RB, Morin PJ. Claudin-containing exosomes in the peripheral circulation of women with ovarian cancer. BMC Cancer. 2009;9:244.

41. Logozzi M, et al. High levels of exosomes expressing CD63 and caveolin-1 in plasma of melanoma patients. PLoS One. 2009;4(4):e5219.

42. Nilsson J, et al. Prostate cancer-derived urine exosomes: a novel approach to biomarkers for prostate cancer. $\mathrm{Br} \mathrm{JCancer}$. 2009;100(10):1603-1607.

43. Skog J, et al. Glioblastoma microvesicles transport RNA and proteins that promote tumour growth and provide diagnostic biomarkers. $\mathrm{Nat}$ Cell Biol. 2008;10(12):1470-1476.
44. Rabinowits G, Gercel-Taylor C, Day JM, Taylor DD, Kloecker GH. Exosomal microRNA: a diagnostic marker for lung cancer. Clin Lung Cancer. 2009;10(1):42-46.

45. Tanaka Y, et al. Clinical impact of serum exosomal microRNA-21 as a clinical biomarker in human esophageal squamous cell carcinoma. Cancer. 2013;119(6):1159-1167.

46. Long JD, et al. A non-invasive miRNA based assay to detect bladder cancer in cell-free urine. Am J Transl Res. 2015;7(11):2500-2509.

47. Li Z, et al. Exosomal microRNA-141 is upregulated in the serum of prostate cancer patients. Onco Targets Ther. 2016;9:139-148.

48. Pfeffer SR, et al. Detection of Exosomal miRNAs in the Plasma of Melanoma Patients. JClin Med. 2015;4(12):2012-2027.

49. Thakur BK, et al. Double-stranded DNA in exosomes: a novel biomarker in cancer detection. Cell Res. 2014;24(6):766-769.

50. San Lucas FA, et al. Minimally invasive genomic and transcriptomic profiling of visceral cancers by next-generation sequencing of circulating exosomes [published online ahead of print December 17, 2015]. Ann Oncol. doi:10.1093/ annonc/mdv604.

51. Lee TH, Chennakrishnaiah S, Audemard E, Montermini L, Meehan B, Rak J. Oncogenic rasdriven cancer cell vesiculation leads to emission of double-stranded DNA capable of interacting with target cells. Biochem Biophys Res Commun. 2014;451(2):295-301.

52. Al-Nedawi K, et al. Intercellular transfer of the oncogenic receptor EGFRvIII by microvesicles derived from tumour cells. Nat Cell Biol. 2008;10(5):619-624.

53. Demory Beckler M, et al. Proteomic analysis of exosomes from mutant KRAS colon cancer cells identifies intercellular transfer of mutant KRAS. Mol Cell Proteomics. 2013;12(2):343-355.

54. Thery C, Duban L, Segura E, Veron P, Lantz O, Amigorena S. Indirect activation of naive $\mathrm{CD} 4$ $\mathrm{T}$ cells by dendritic cell-derived exosomes. Nat Immunol. 2002;3(12):1156-1162.

55. Greening DW, Gopal SK, Xu R, Simpson RJ, Chen W. Exosomes and their roles in immune regulation and cancer. Semin Cell Dev Biol. 2015;40:72-81.

56. Clayton A, Mason MD. Exosomes in tumour immunity. Curr Oncol. 2009;16(3):46-49.

57. Zitvogel L, et al. Eradication of established murine tumors using a novel cell-free vaccine: dendritic cell-derived exosomes. Nat Med. 1998;4(5):594-600.

58. Hwang I, Shen X, Sprent J. Direct stimulation of naive $\mathrm{T}$ cells by membrane vesicles from antigen-presenting cells: distinct roles for CD54 and B7 molecules. Proc Natl Acad Sci U S A. 2003;100(11):6670-6675.

59. André F, et al. Tumor-derived exosomes: a new source of tumor rejection antigens. Vaccine. 2002;20(suppl 4):A28-A31.

60. Andre F, et al. Malignant effusions and immunogenic tumour-derived exosomes. Lancet. 2002;360(9329):295-305.

61. Schartz NE, Chaput N, Andre F, Zitvogel L. From the antigen-presenting cell to the antigenpresenting vesicle: the exosomes. Curr Opin Mol Ther. 2002;4(4):372-381.
62. Wolfers J, et al. Tumor-derived exosomes are a source of shared tumor rejection antigens for CTL cross-priming. Nat Med. 2001;7(3):297-303.

63. Lancaster GI, Febbraio MA. Exosome-dependent trafficking of HSP70: a novel secretory pathway for cellular stress proteins. J Biol Chem. 2005;280(24):23349-23355.

64. Skokos D, et al. Mast cell-derived exosomes induce phenotypic and functional maturation of dendritic cells and elicit specific immune responses in vivo. JImmunol. 2003;170(6):3037-3045.

65. Yu S, et al. Tumor exosomes inhibit differentiation of bone marrow dendritic cells. JImmunol. 2007;178(11):6867-6875

66. Clayton A, Mitchell JP, Court J, Linnane S, Mason MD, Tabi Z. Human tumor-derived exosomes down-modulate NKG2D expression. JImmunol. 2008;180(11):7249-7258.

67. Andreola $G$, et al. Induction of lymphocyte apoptosis by tumor cell secretion of FasL-bearing microvesicles. J Exp Med. 2002;195(10):1303-1316.

68. Soderberg A, Barral AM, Soderstrom M, Sander $\mathrm{B}$, Rosen A. Redox-signaling transmitted in trans to neighboring cells by melanoma-derived TNF-containing exosomes. Free Radic Biol Med. 2007;43(1):90-99.

69. Taylor DD, Gercel-Taylor C. Tumour-derived exosomes and their role in cancer-associated T-cell signalling defects. Br JCancer. 2005;92(2):305-311.

70. Muller L, Mitsuhashi M, Simms P, Gooding WE, Whiteside TL. Tumor-derived exosomes regulate expression of immune function-related genes in human T cell subsets. Sci Rep. 2016;6:20254.

71. Clayton A, Mitchell JP, Court J, Mason MD, Tabi Z. Human tumor-derived exosomes selectively impair lymphocyte responses to interleukin-2. Cancer Res. 2007;67(15):7458-7466

72. Morse MA, et al. A phase I study of dexosome immunotherapy in patients with advanced non -small cell lung cancer. J Transl Med. 2005;3(1):9.

73. Ribeiro MF, Zhu H, Millard RW, Fan GC. Exosomes function in pro- and anti-angiogenesis. Curr Angiogenes. 2013;2(1):54-59.

74. Umezu T, Ohyashiki K, Kuroda M, Ohyashiki $\mathrm{JH}$. Leukemia cell to endothelial cell communication via exosomal miRNAs. Oncogene. 2013;32(22):2747-2755

75. Park JE, et al. Hypoxic tumor cell modulates its microenvironment to enhance angiogenic and metastatic potential by secretion of proteins and exosomes. Mol Cell Proteomics. 2010;9(6):1085-1099.

76. Hong BS, et al. Colorectal cancer cell-derived microvesicles are enriched in cell cycle-related mRNAs that promote proliferation of endothelial cells. BMC Genomics. 2009;10:556.

77. Kucharzewska P, et al. Exosomes reflect the hypoxic status of glioma cells and mediate hypoxia-dependent activation of vascular cells during tumor development. Proc Natl Acad Sci U S A. 2013;110(18):7312-7317.

78. Zhou W, et al. Cancer-secreted miR-105 destroys vascular endothelial barriers to promote metastasis. Cancer Cell. 2014;25(4):501-515.

79. Webber JP, et al. Differentiation of tumour-promoting stromal myofibroblasts by cancer exosomes. Oncogene. 2015;34(3):290-302.

80. Webber J, Steadman R, Mason MD, Tabi Z, 
Clayton A. Cancer exosomes trigger fibroblast to myofibroblast differentiation. Cancer Res. 2010;70(23):9621-9630.

81. Cho JA, Park H, Lim EH, Lee KW. Exosomes from breast cancer cells can convert adipose tissuederived mesenchymal stem cells into myofibroblast-like cells. Int JOncol. 2012;40(1):130-138.

82. Hu Y, et al. Fibroblast-derived exosomes contribute to chemoresistance through priming cancer stem cells in colorectal cancer. PLoS One. 2015;10(5):e0125625.

83. Boelens MC, et al. Exosome transfer from stromal to breast cancer cells regulates therapy resistance pathways. Cell. 2014;159(3):499-513.

84. Abd Elmageed ZY, et al. Neoplastic reprogramming of patient-derived adipose stem cells by prostate cancer cell-associated exosomes. Stem Cells. 2014;32(4):983-997.

85. Antonyak MA, et al. Cancer cell-derived microvesicles induce transformation by transferring tissue transglutaminase and fibronectin to recipient cells. Proc Natl Acad Sci U S A. 2011;108(12):4852-4857.

86. Hoshino D, et al. Exosome secretion is enhanced by invadopodia and drives invasive behavior. Cell Rep. 2013;5(5):1159-1168.
87. Mu W, Rana S, Zoller M. Host matrix modulation by tumor exosomes promotes motility and invasiveness. Neoplasia. 2013;15(8):875-887.

88. Le MT, et al. miR-200-containing extracellular vesicles promote breast cancer cell metastasis. JClin Invest. 2014;124(12):5109-5128.

89. Vella LJ. The emerging role of exosomes in epithelial-mesenchymal-transition in cancer. Front Oncol. 2014;4:361.

90. Hoshino A, et al. Tumour exosome integrins determine organotropic metastasis. Nature. 2015;527(7578):329-335.

91. Kosaka N, Iguchi H, Hagiwara K, Yoshioka Y, Takeshita F, Ochiya T. Neutral sphingomyelinase 2 (nSMase2)-dependent exosomal transfer of angiogenic microRNAs regulate cancer cell metastasis. J Biol Chem. 2013;288(15):10849-10859.

92. Yin J, et al. Secretion of annexin A3 from ovarian cancer cells and its association with platinum resistance in ovarian cancer patients. J Cell Mol Med. 2012;16(2):337-348.

93. Federici $\mathrm{C}$, et al. Exosome release and low $\mathrm{pH}$ belong to a framework of resistance of human melanoma cells to cisplatin. PLoS One. 2014;9(2):e88193.
94. Shedden K, Xie XT, Chandaroy P, Chang YT, Rosania GR. Expulsion of small molecules in vesicles shed by cancer cells: association with gene expression and chemosensitivity profiles. Cancer Res. 2003;63(15):4331-4337.

95. Marleau A, Chen C-S, Joyce J, Tullis R. Exosome removal as a therapeutic adjuvant in cancer. J Transl Med. 2012;10:134.

96. Ichim TE, et al. Exosomes as a tumor immune escape mechanism: possible therapeutic implications. J Transl Med. 2008;6:37.

97. Vader P, Breakefield XO, Wood MJ. Extracellular vesicles: emerging targets for cancer therapy. Trends Mol Med.2014;20(7):385-393.

98. Johnsen KB, Gudbergsson JM, Skov MN, Pilgaard L, Moos T, Duroux M. A comprehensive overview of exosomes as drug delivery vehicles - endogenous nanocarriers for targeted cancer therapy. Biochim Biophys Acta. 2014;1846(1):75-87.

99. Cooper JM, et al. Systemic exosomal siRNA delivery reduced alpha-synuclein aggregates in brains of transgenic mice. Mov Disord. 2014;29(12):1476-1485.

100.Ohno S, et al. Systemically injected exosomes targeted to EGFR deliver antitumor microRNA to breast cancer cells. Mol Ther. 2013;21(1):185-191. 